\title{
A PATHOLOGICAL STUDY OF EMPHYSEMA OF THE LUNGS WITH CHRONIC BRONCHITIS
}

\author{
BY \\ BRIAN E. HEARD \\ From the Department of Morbid Anatomy, Postgraduate Medical School of London
}

(RECEIVED FOR PUBLICATION DECEMBER 20, 1957)

The terms chronic bronchitis and emphysema have been used together for many years, but unless the emphysema is advanced there can be no clinical or radiological proof of its existence in a "bronchitic." Even in necropsy material the presence of centrilobular emphysema has been identified only very recently (Gough, 1952 ; McLean, 1956a ; Leopold and Gough, 1957). Fletcher (1952) points out that the objective methods of the physiologist can help the clinician in his perplexity over the clinical diagnosis of emphysema. Further work on the basic pathological appearances of emphysema should be of some assistance in interpreting physiological findings in chronic bronchitics.

It was decided, on these grounds, to examine a series of lungs obtained at necropsy from patients with clinical evidence of chronic bronchitis, using special methods to demonstrate emphysema. The series is small and growing slowly, but since 12 of the first 14 cases showed widespread centrilobular emphysema, it was thought worth while to describe the methods and some of the findings to date. A close clinico-pathological study is the eventual aim of the work.

The clinical features of chronic bronchitis have been summarized by Scadding (1952) and by Oswald, Harold, and Martin (1953). Selection of the present cases was based on their descriptions.

Pathologists have used unreliable signs of emphysema in the past. Bullae are not always an indication of widespread disease and may be seen quite commonly in the upper lobes of elderly subjects with otherwise relatively normal lungs. Bullae may be absent from lungs showing widespread centrilobular emphysema. Large lungs bulging from the opened thorax at necropsy indicate terminal bronchial obstruction which may mean acute emphysema rather than chronic. A more reliable indication of emphysema is the soft, stringy character of the cut surface of the severely affected lung, though this is often difficult to see. Moderate grades can be found by floating slices of lung in water and this is a useful procedure, but the translucency of lung tissue still prevents fine details from being recognized with conviction.

The translucency of the lung can be overcome by a simple method, described here, of impregnating lung slices with barium sulphate. Fortunately, centrilobular lesions affect dust-pigmented respiratory bronchioles, and these dark dilated bronchioles contrast well with the barium-whitened surroundings. The procedure can be carried out on unfixed, or quickly-fixed, tissue at the time of necropsy. It is best applied to material fixed by distension of the bronchial tree with formalin. The procedure greatly facilitates photography, as the accompanying figures illustrate.

The first essential for a reliable estimate of emphysema is fixation of the lung with even, though gentle, distension of all air spaces. This has been achieved by prolonging the period of fixation under pressure. A simple device is described for applying a continuous pressure of $10 \mathrm{in}$. $(25 \mathrm{~cm}$.) of formalin for days or weeks at a time, and barium impregnation of slices of such material gives reliable results.

Some recent specimens have been injected through the pulmonary arteries with a bariumgelatin mixture by Professor C. V. Harrison. This method shows up the fine lobular branches of the pulmonary arteries in lung slices and demonstrates the centrilobular distribution of lesions where septa are ill defined (Fig. 8). It also shows that in advanced diffuse emphysema the arteries remain intact (Fig. 4), while the other structures of the lobule atrophy.

Focal emphysema has been described by Gough (1940) and Heppleston (1947). Recent work on non-industrial emphysema began in 1948 with the development by Gough and Wentworth of the now well-known method of entire-lung sections. 
In 1952 Gough divided emphysema into two varieties, one in which forces appeared to affect the lung as one unit and the other in which forces appeared to affect the secondary lobules. In 1957 Leopold and Gough gave a full account of centrilobular emphysema, describing it as a frequent complication of the generalized form. Right ventricular hypertrophy was present in only nine out of 65 cases of generalized emphysema, but was seen in 41 out of 75 cases with added centrilobular emphysema.

Spain and Kaufman (1953) examined multiple sections of emphysematous lungs and described changes in bronchioles which they considered to be the basic lesion of emphysema.

Reid $(1954,1955,1956)$ applied the serial section technique to the study of lung nodules in chronic bronchitis and demonstrated obliteration or dilatation of small bronchioles together with alveolar changes. Bronchiolar changes were sometimes located in specimens by injecting the bronchi with radio-opaque material.

McLean (1956a) used several methods to study emphysema. He fixed some lungs by distending the air passages with formalin, and others by the intravenous injection of formalin into the cadaver. He examined part of his material by the Gough and Wentworth technique, but he also examined and photographed lung slices. Emphysema was classified into localized and generalized forms and the latter was further subdivided into centrilobular and diffuse forms (see original article for full classification). Examples of these were photographed under water with careful attention to lighting arrangements, and the photographs of the present article are similar to his, but show more detail after barium sulphate impregnation. In later papers McLean (1956b, 1957a, b, and c) attributed the bronchiolectasis of centrilobular emphysema to a proximal bronchiolitis and airtrapping.

\section{FixATION METHOD}

All ordinary precautions were taken to avoid cutting or tearing the lungs as they were removed. If adhesions were dense, extrapleural dissection was carried out. Small cuts were sutured before fixation. The routine practice of running a pair of scissors down the membranous posterior wall of the trachea was done with care, in order to preserve sufficient main bronchus to take a cannula. Where delay was inevitable, the lung was covered with a damp cloth to prevent the pleural surface from drying.

The segmental bronchi were sucked clear of mucus with a narrow glass cannula. A broad cannula was then tied tightly into the main bronchus with string.
A rubber bung, with a circumferential groove, mounted broad end distally on glass tubing, was found ideal (Harrison, 1955) and a selection of various sizes of these was kept available. If an important specimen had been cut near the first bronchial division, smaller cannulae were tied into the main upper and lower lobe bronchi and supplied by a glass Y-piece.

The lung was floated in water and distended gently with $20 \%$ formol saline at a pressure of $10-12$ in. $(25-30 \mathrm{~cm}$.) of fixative. When fully distended it was immersed in fixative and attached to the apparatus to be described. If a segment failed to fill after several minutes the pressure was briefly raised to 36 in. $(90 \mathrm{~cm}$.) until the obstruction was overcome.

The usual practice with lungs which have been treated in this way is to seal off the bronchus and allow the lung to float in formalin. I have examined numerous specimens fixed in this way and have had no difficulty in mapping out coarser grades of emphysema, e.g., as seen in Fig. 5, but with the finer grades of change errors may appear. The floating lung flattens and shrinks as the formalin leaks away through the pulmonary vessels. Formalin is a slow fixative and I have found that shrinkage can be prevented only by maintaining the pressure of the injection at 10 to 12 in. for several days, preferably two weeks. There is no significant change in the volume of the lung over the fixation period nor for weeks or months after the pressure has been released. The lung retains its anatomical features precisely and slices show even fixation.

The main parts of the apparatus are illustrated in Fig. 1. The head of pressure is supplied through a syphon from the flask $D$, the upper end of the overflow $\mathrm{H}$ being set $10-12$ in. $(25-30 \mathrm{~cm}$.) above the fluid level in the lung container $F$. Continuous suction is applied at G, drawing fixative into the upper container A. When the level of fluid in the lung container drops below the lower end of the tube E, the vacuum breaks and fixative flows past the one-way glass valve $C$ into flask $D$. Excess fluid escapes into the lung container by the overflow $\mathrm{H}$, restoring the vacuum. The glass valve $C$ prevents fixative from being drawn up from the flask $D$ to the upper container $\mathrm{A}$, and is made to carry a fast downward flow of fixative when the vacuum breaks. The clip $B$ is adjusted to prevent the flask from overflowing.

Suction is applied in two ways. While the lung is filling, a filter pump (Edwards's "speedivac" metal water jet pump) is used to cope with the large volume of fixative required. After 30 minutes this can be replaced by an oil pump with an induction motor of the type used surgically to apply continuous suction to pleural sacs, etc. (Roberts's suction pump). The time take for this slower pump to fill the upper container leads to a desirable intermittency and the cycle recurs every two minutes approximately. Although a simple water pump would refill the reservoir efficiently, there appears to be no pump available for dealing quietly with small volumes of fluid. The present apparatus can be left to run without attention 


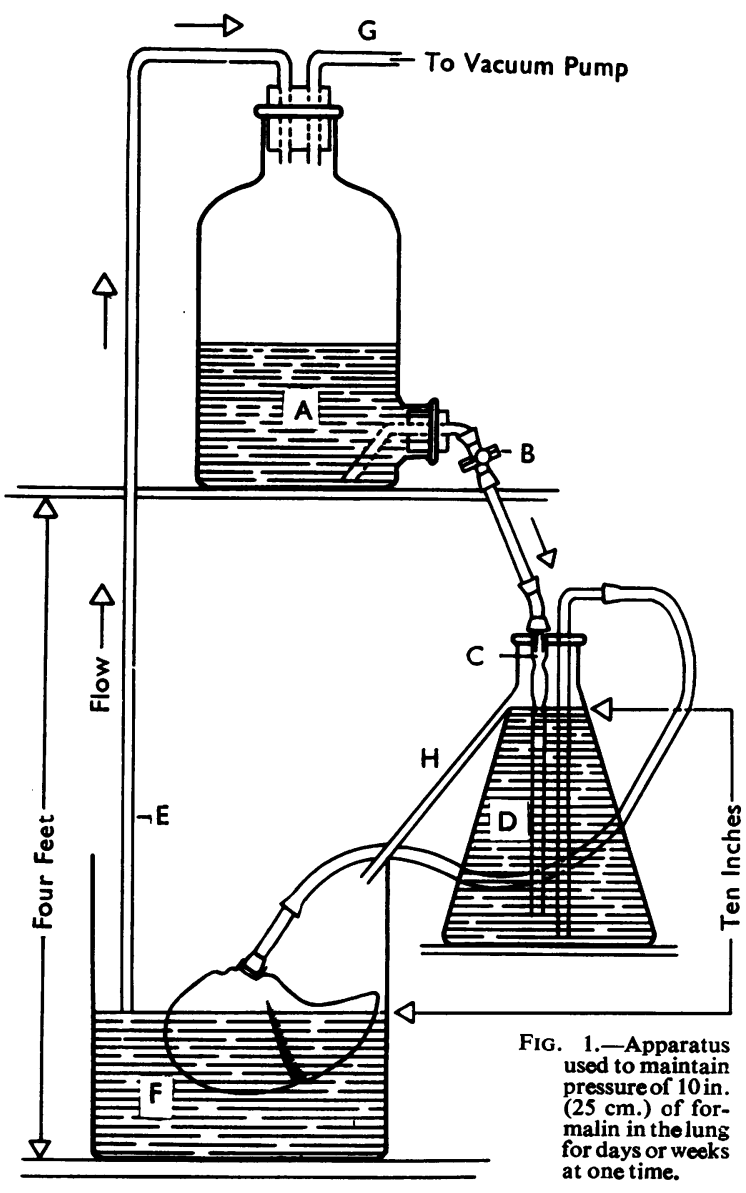

for several days at a time, and a vacuum pump of the above type is subjected to far less wear and tear than would be suffered by a positive-pressure water pump. The apparatus functions well with a filter pump and the oil pump is necessary only if many lungs are to be studied. Several lungs can be fixed at one time in a larger lung container, supplied in parallel from the flask $D$.

The fixative chosen was $20 \% \quad \mathrm{v} / \mathrm{v}$ aqueous formalin; colour preservatives and buffers were omitted. The strength of the formalin was higher than usual to speed fixation and to allow for formalin vapour extracted by the vacuum.

Fixation under pressure was continued for as long as possible, usually one to two weeks. A minimum of three days was essential. Gough and Wentworth (1948) found enzymes to be active after prolonged formalin fixation, so the longest fixation periods possible are recommended.

After long fixation the lung lies lower in the fixative and may sink. At the moment, in an attempt to remove air bubbles, I am inflating the lungs with carbon dioxide several times before distension with fixative (Tompsett, 1952).
Barium Sulphate Impregnation Method

The fixed lung was sliced on a board fitted with rails $0.8 \mathrm{~cm}$. high to support each end of the $14 \mathrm{in}$. $\frac{\bar{\sigma}}{2}$ $(35 \mathrm{~cm}$.) blade of a ham knife (Cunningham and $\vec{\varnothing}$ Miller, 1952). The lung was cut with the costal $\varrho$ surface lowermost and slices then ran antero- $\%$ posteriorly and included the main segments. Severely $\vec{\circ}$ emphysematous lungs cut well if the pressure of formalin was maintained through the bronchi as the knife passed through the lung.

Barium impregnation was devised to render the $\vec{x}$ translucent lung tissue opaque. A selected slice $\stackrel{x}{\vec{\omega}}$ lateral to the main bronchi was squeezed lightly in $\vec{\omega}$ water and placed in a tray of warm, aqueous barium ? nitrate (approximately $75 \mathrm{~g}$. in 1 litre of water). The $\vec{\omega}$ lung was gently pressed in all parts to aid absorption o of the solution and, after one minute, the slice was 9 lightly squeezed out and transferred to a warm aqueous solution of sodium sulphate (approximately $\check{\complement}$ $100 \mathrm{~g}$. in 1 litre of water). The reagents were stored in an incubator at $37^{\circ} \mathrm{C}$. and were thus available for use at any time. The lung slice was again pressed in $\mathcal{E}$ all areas for one minute, squeezed out lightly and 90 returned to the barium nitrate. This was repeated two to four times and the whitened slice was squeezed lightly in water and stored in a large vessel of $20 \%$ formalin. Lung slices became distorted permanently if they were not stored flat. The barium sulphate impregnation usually increased the weight and caused them to sink slowly in the fixative. Up to 12 slices could be stored flat resting lightly one upon the other without becoming distorted.

Barium sulphate was precipitated very finely on all structures and did not detach readily. The treated slices were examined in trays under water. They were greyish-white and no attempt was made to bring back the original colour. Dust pigment was not obscured by moderate barium sulphate impregnation, and since centrilobular emphysema affects the frequently pigmented respiratory bronchioles, these showed up as dark holes against white unaffected lung - a great aid to visualization and to photography (with the slice in water). The finer details, as viewed in water by means of a dissecting microscope, were extremely satisfactory (see Fig. 4). The method has been used on slices of fresh tissue, fixed quickly by floating flat in of strong formalin for one half to one hour, to demonstrate major findings urgently. It has also been used on museum preparations of emphysematous lungs.

\section{RESULTS}

Lungs are being taken at necropsy from patients with a history of chronic bronchitis and compared with a series from patients with non-respiratory illnesses. From the study of this material to date, and in the light of recent work quoted in the introduction, the following anatomical classification of emphysema has been framed : (1) Diffuse emphysema, and (2) centrilobular emphysema. Each of 8 these may be $(a)$ widespread or $(b)$ localized. 


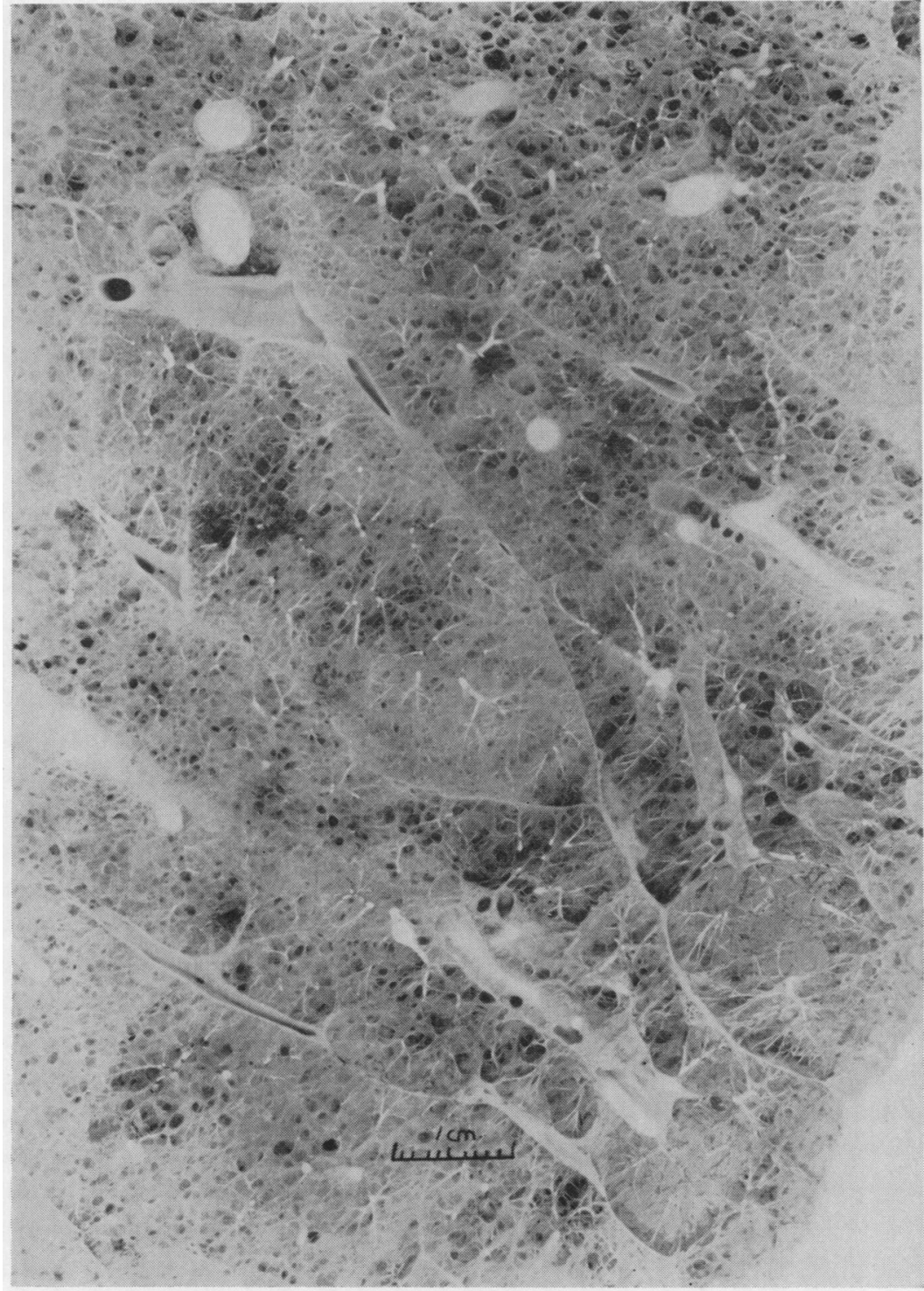

FIG. 2.-Widespread diffuse emphysema ( $\times 1 \cdot 4)$.

I have not used the term " generalized," because by this McLean (1956a) denoted the extent of either diffuse or centrilobular forms, while Leopold and Gough (1957) denoted one form only (diffuse). Further confusion arises when diffuse emphysema is localized, for if it were termed "generalized" the local form would have to be called "localized generalized emphysema."

The above classification needs to be extended in certain instances. Many routine specimens from elderly patients have bullae on the medial aspect of the upper lobe while the rest of the lung shows very little change. This is termed "superficial emphysema" here. Occasionally the periphery of a lobule is stretched from the septum (Fig. 13) and I have used the term " paraseptal emphysema" for this. A minor complication is that the layer of localized emphysema around scars may affect only parts of lobules, but this can be included in the term "localized diffuse emphysema" for convenience. Tension cysts (McLean, 1956a) can be labelled as such. "Senile" emphysema can probably be described as a mild

The two main divisions rest on an appreciation of the lobular structure of the lung. In diffuse emphysema there may be changes in all parts of the secondary lobule, while in centrilobular emphysema the changes are mainly confined to the middle parts. Frequently both varieties occurred in the same lung and were recorded separately. The extent of the lesions was indicated clearly by the terms widespread and localized. widespread diffuse emphysema of later life. Interstitial emphysema is outside the present grouping, as also are honeycomb and cystic lungs. Emphysematous cysts are recorded separately, though they may be part of a widespread emphysema. "Localized hypertrophic emphysema," which occurs mainly in children (Silver, Kirklin, Harris, and McDonald, 1956), can be grouped anatomically as a form of localized diffuse emphysema. 
WidESPREAD DiffUSE EMPHYSEMA. - This is illustrated in Figs. 2-4. This is the left lung from a man of 55 who had chronic bronchitis for 20 years and died of cor pulmonale.* The left lower lobe was partially collapsed and scarred from an attack of pneumonia seven years before death. Not one normal secondary lobule was seen anywhere in either lung. Fig. 2 shows details of the upper lingular segment which was less affected than other segments. Most lobules, even here, have lost the normal dense detail and are composed of large air spaces with fairly thick walls. This change often involves peripheral lobular tissue. The $\mathrm{V}$-shaped lobule in the centre may appear at first sight to be relatively normal, but by the dissecting microscope it is seen to be made up of soft, thin, atrophic walls of the type more often seen in localized diffuse emphysema.

The pulmonary arteries were injected with a fine barium and gelatin mixture before fixation. In Fig. 3 is an atrophic lobule picked from the anterior apical segment. The appropriate branch of the pulmonary artery enters the lobule on the left and, as a result of atrophy of the walls of air spaces, can be followed to ramifications at precapillary level. In Fig. 4 the arteries are seen to be almost the last remaining structures of the lobule apart from a few residual lightly pigmented strands of atrophic lung stretched across the interarterial gaps. A few capillaries are filled with injected barium.

The predominant change in this lung, however, is the loss of fine alveolar structure and its replace-

ment by relatively large, thick-walled spaces-aN widespread diffuse emphysema of advanced N degree.

Centrilobular Emphysema.-This is a forme affecting more central respiratory bronchioles (a亦 bronchiolectasis). Fig. 5 shows these dilated bronchioles lying in clusters well away from the inter-0 lobular septa. This lung was from a man of $51 \mathbb{\Phi}$ years who had chronic bronchitis for 10 years, $\frac{\mathcal{P}}{\mathbb{Q}}$ following an attack of pneumonia. He died of $\frac{\varrho}{Q}$ cor pulmonale (and Ellis' type II nephritis).

Fig. 6 shows part of the apical segment of theo left lower lobe of the lung of a man of 58 years from case to case. 
FIG. 4,-Higher magnification of Fig. 3 ( $\times 10)$.

FIG. 5.-Widespread centrilobular emphysema $(\times 3)$.

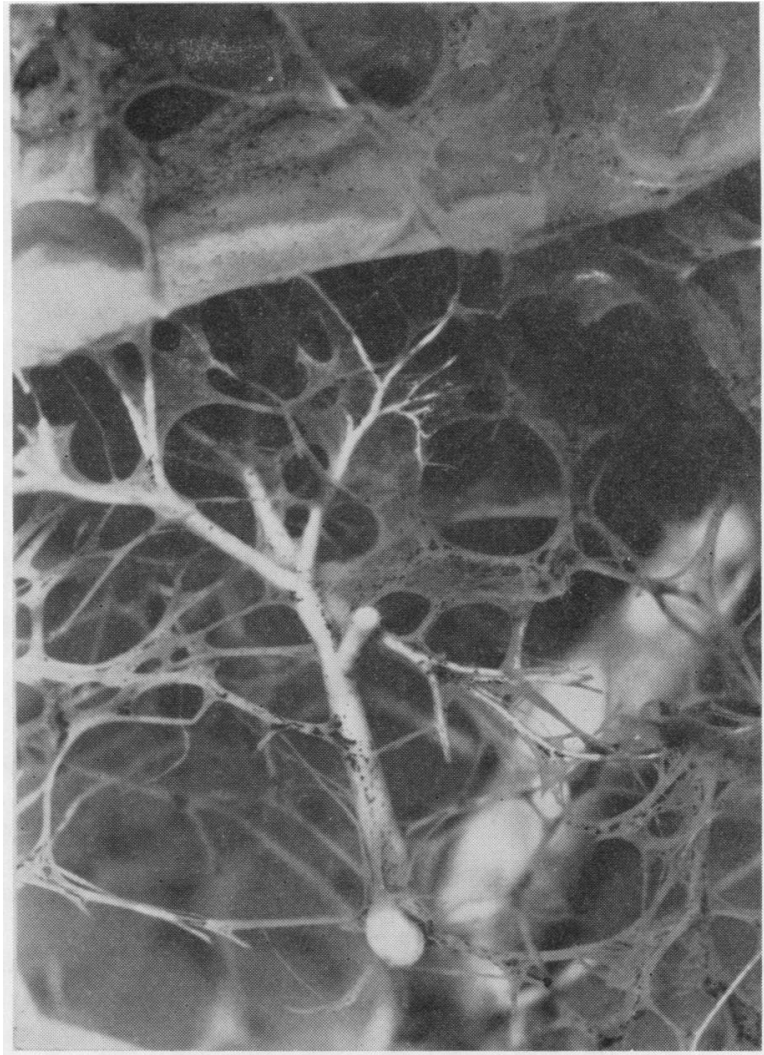

Fio. 4

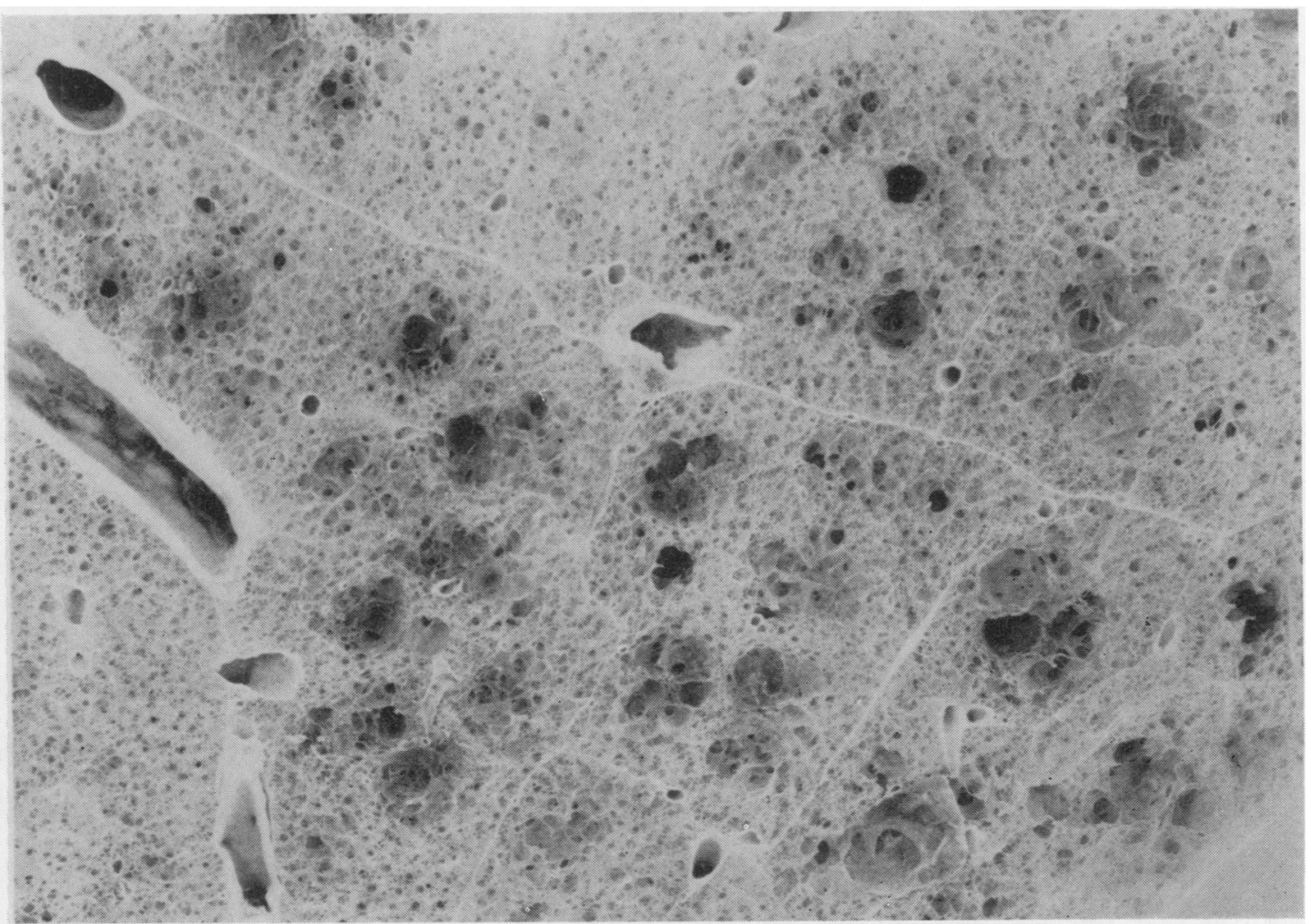




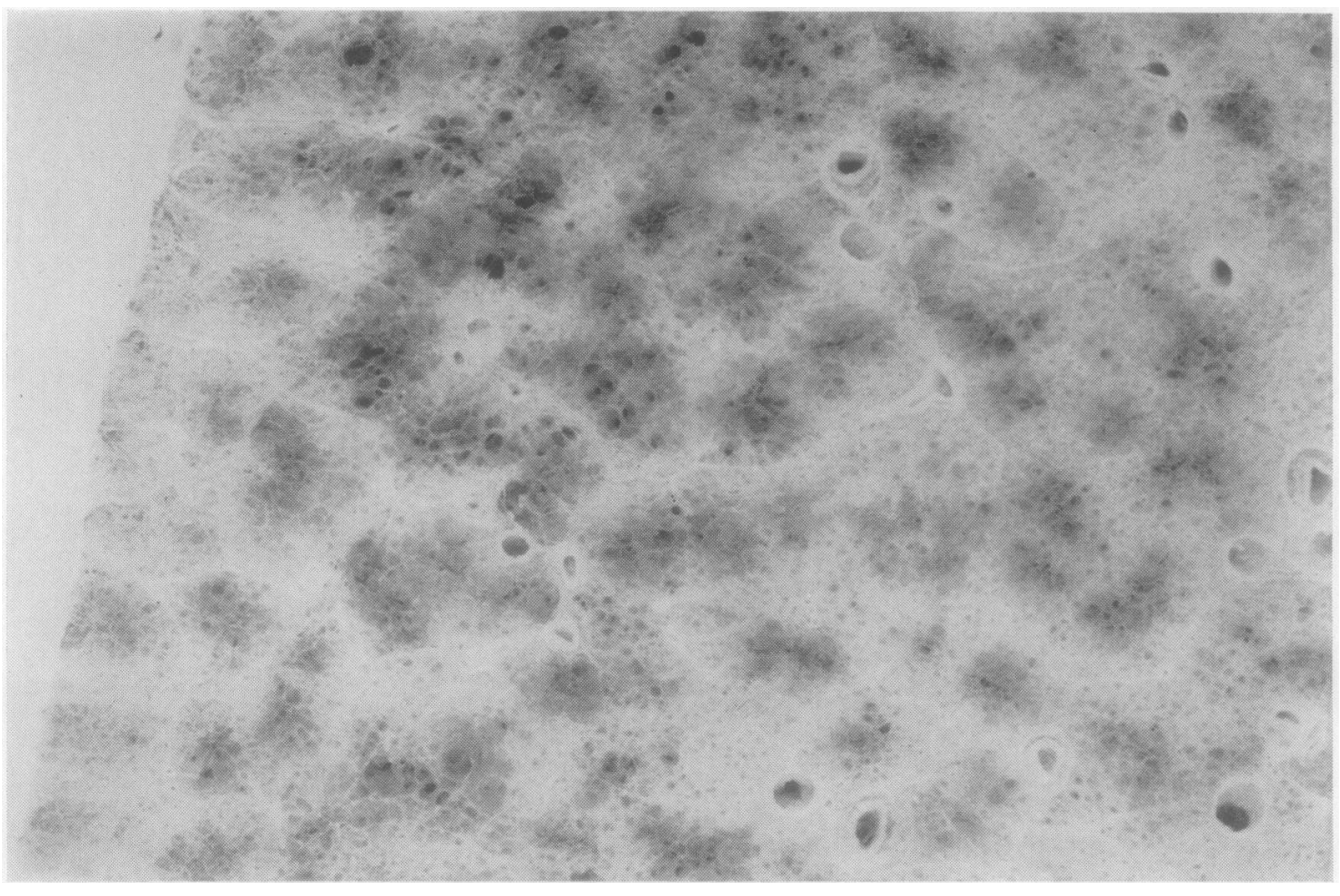

FIG. 6.-Widespread centrilobular emphysema (mild) $(\times 1.75)$.

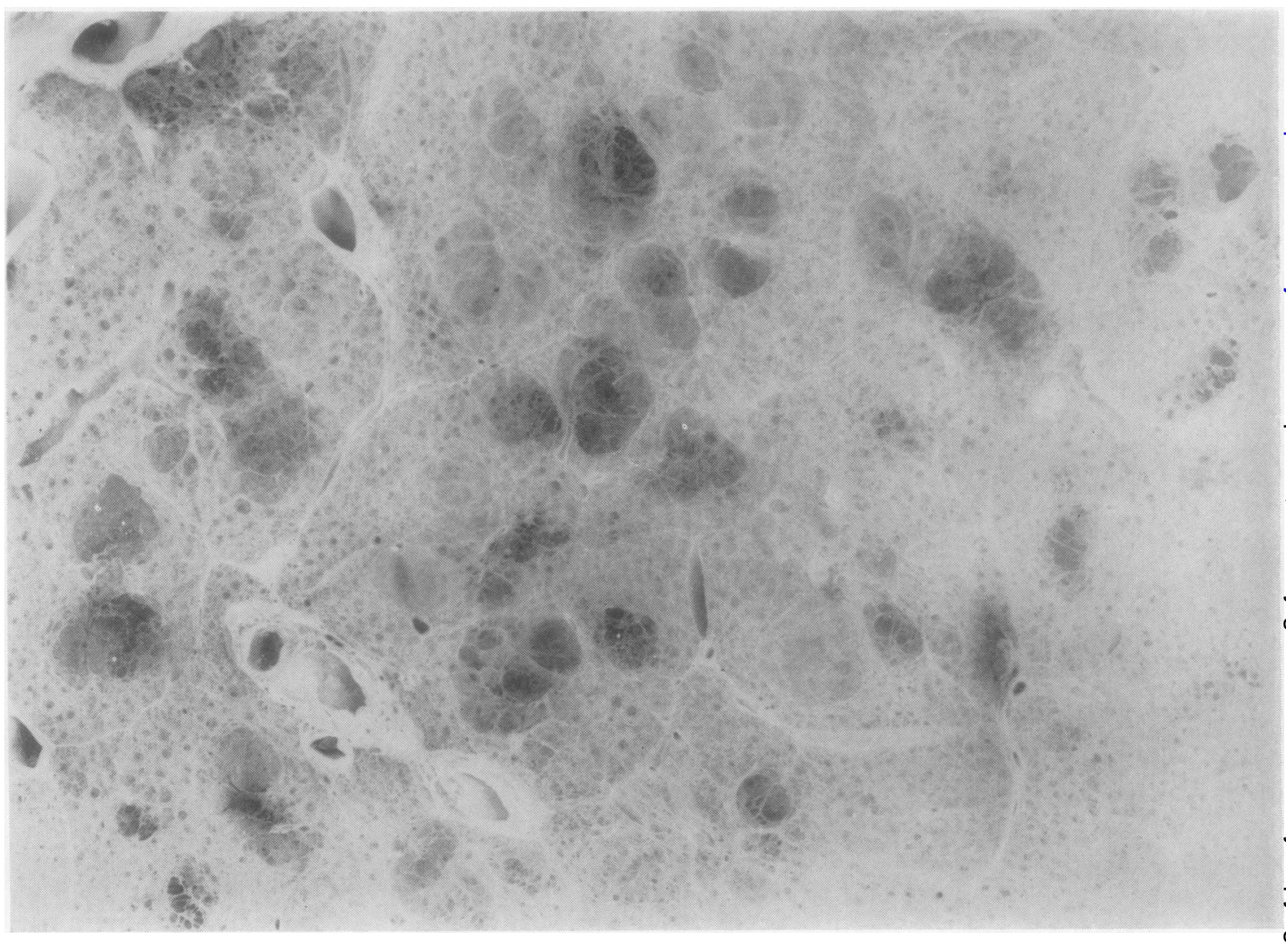

FIG. 7.-Widespread centrilobular emphysema $(\times 2 \cdot 2)$. 


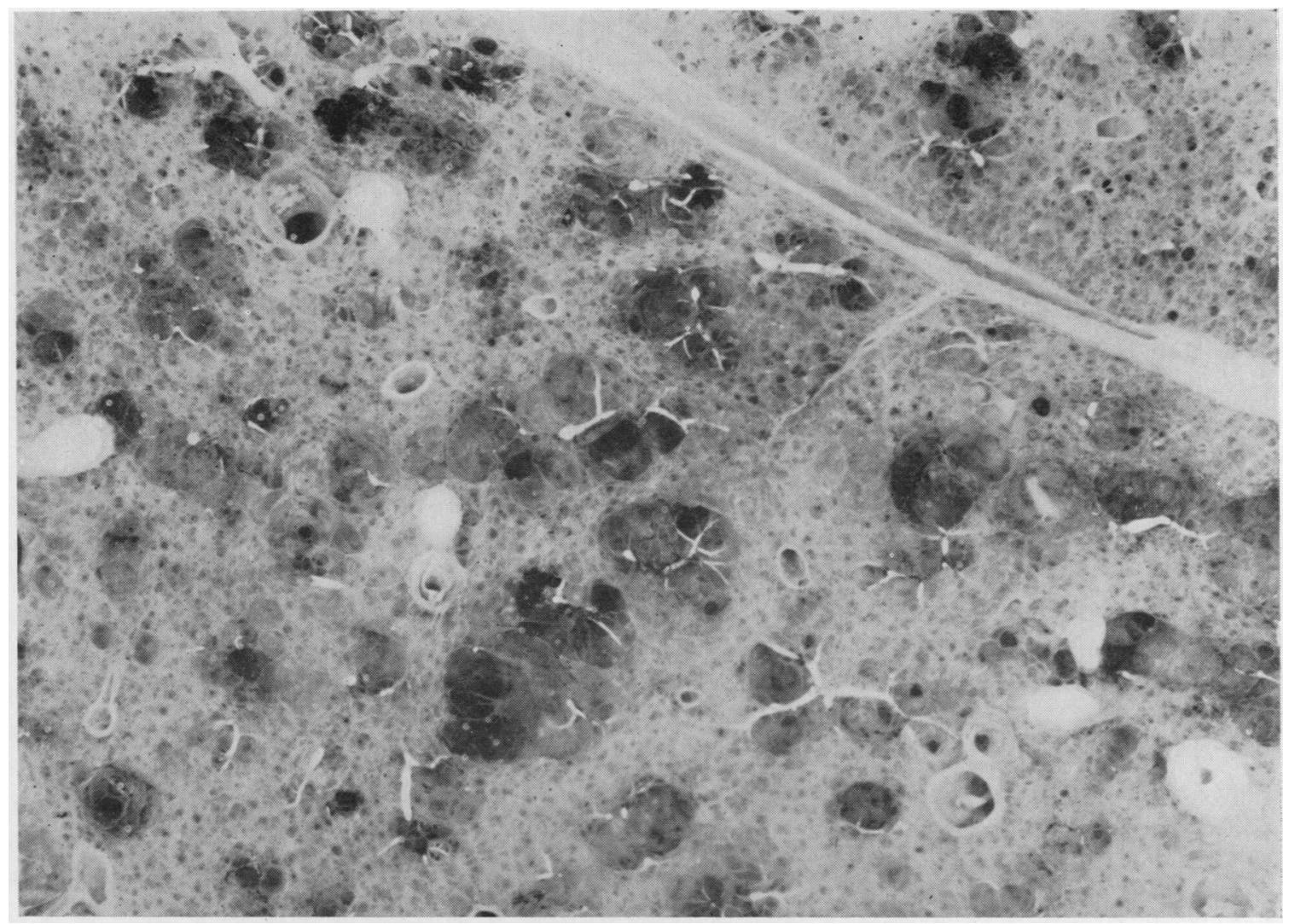

FIG. 8.-Widespread centrilobular emphysema ( $\times 3$ ).

who died of coronary thrombosis. He had " bronchitis" at the age of 6 years, but complained only latterly of cough, worse in winter. He smoked 25 cigarettes a day and stoked furnaces for over 20 years. The lung shows mild, but widespread, centrilobular emphysema. Almost every lobule has a central cluster of slightly dilated, pigmented bronchioles. The lesions were not detected in the fresh right lung at necropsy, but are obvious in the barium-impregnated slices of the long-fixed lung. Control material shows that this is a true centrilobular emphysema and not an artefact produced by distension with formalin. Note the relatively normal lung substance near the interlobular septa.

Fig. 7 is part of the anterior segment of the upper lobe of the right lung from a man of 75 years who died of carcinoma of the left lung. He smoked 60 cigarettes a day from the age of 18 , complained of a " smoker's cough " all his life, had winter bronchitis for "years," more serious for the last five years, and three years before death had had bronchopneumonia. There is more marked centrilobular emphysema than in the previous case and the dilated respiratory bronchioles have run together to form prominent cyst-like spaces crossed by stretched vessels and remnants of bronchiolar walls.

Fig. 8 is part of the lower lobe of the right lung from a man of 55 years who died of cor pulmonale. He had pneumonia 17 years previously and his chronic bronchitis dated from that event. There was post-inflammatory scarring of the upper lobe and widespread bronchiectasis. This slice shows widespread centrilobular emphysema and, in spite of the absence of interlobular septa, the centrilobular position of the lesions is shown by their tendency to surround the terminal branches of the injected pulmonary arterial tree. For the same reason, the lesions avoid the regions of the pulmonary veins.

Fig. 9 is the right lung of a woman of 37 who died of cor pulmonale 13 years after a left-sided thoracoplasty for pulmonary tuberculosis. For the last four years she had chronic bronchitis with 


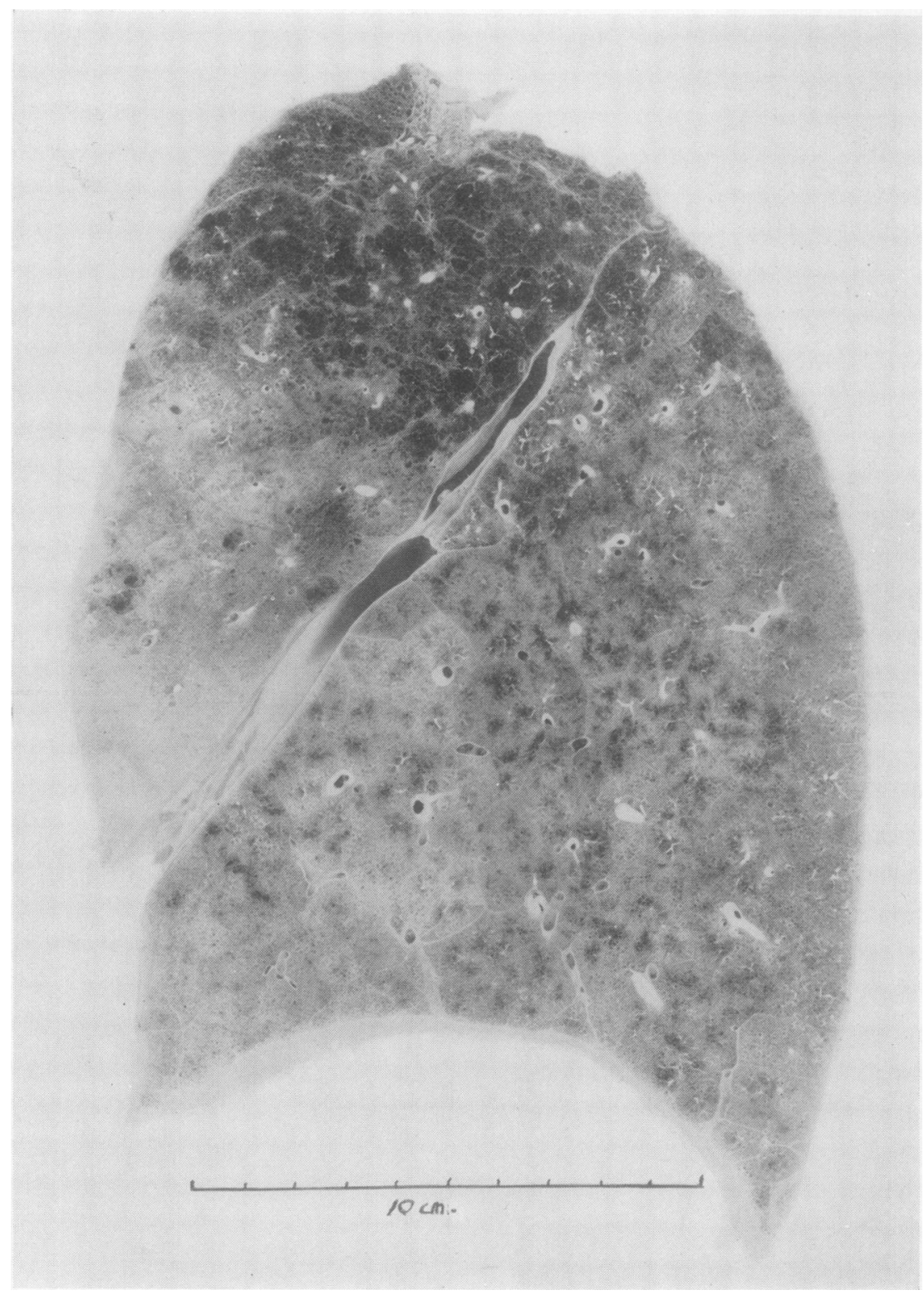

Fig. 9.-Widespread centrilobular emphysema with upper lobe accentuation $(\times 0.63)$.

exacerbations in the winter. The lower lobe shows a mild grade of pigmented centrilobular emphysema in most areas. The middle lobe is almost normal, though the absence of pigment may indicate previous inflammation (Gough, 1957). The upper lobe shows advanced emphysema, heavily dust-pigmented. This is a case of mild widespread centrilobular emphysema with accentuation near the scarring of healed apical tuberculosis.
Fig. 10 shows that the change in the upper lobe is basically centrilobular in type (note the bariumgelatin injection of a number of the lobular arteries).

Mixed patterns were common. Fig. 11 is the left lung of a man of 68 years with a long history of chronic bronchitis dating from gassing in the first world war. He died of cor pulmonale. In the centre of the upper lobe there is marked centri- 


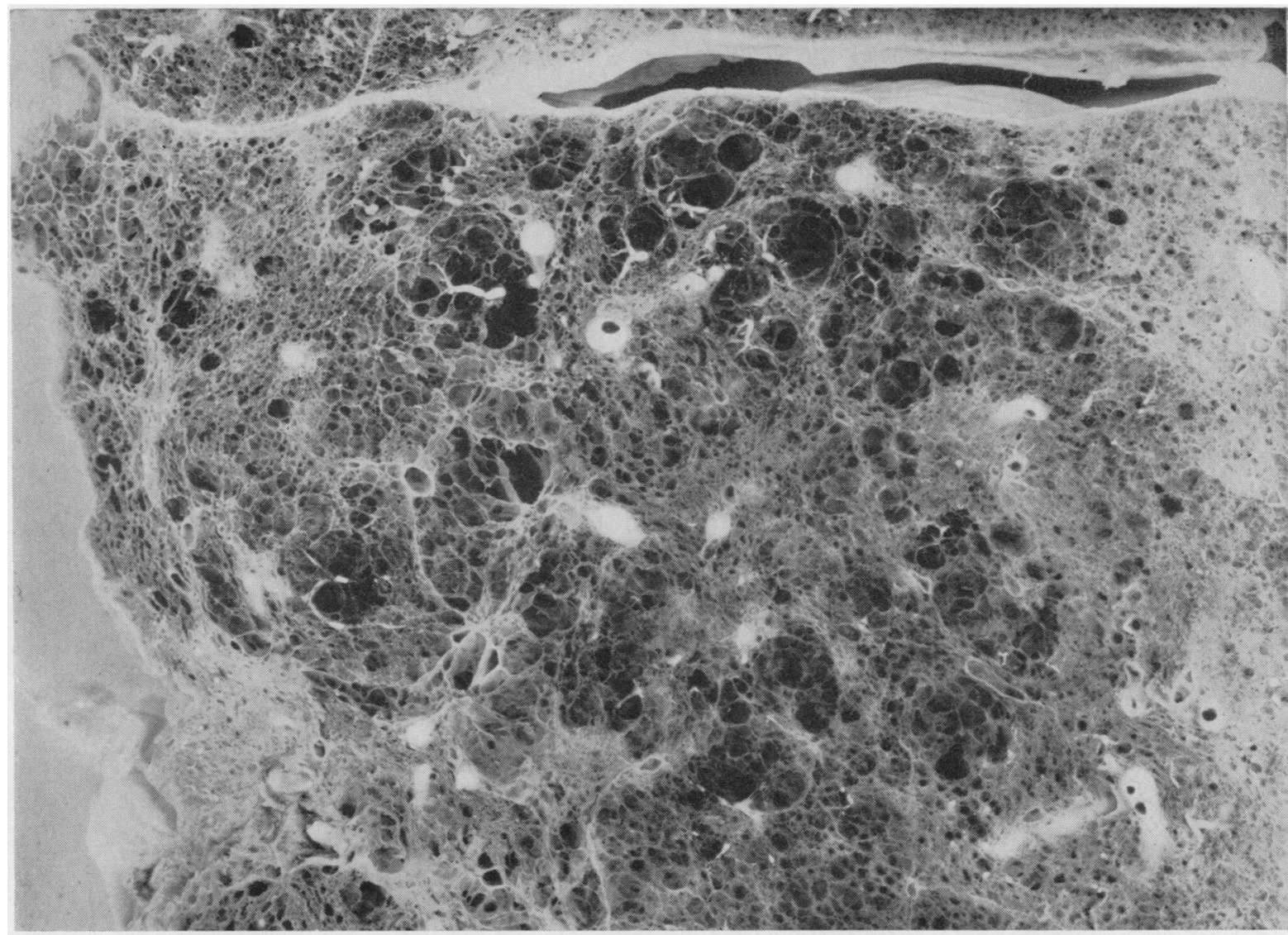

FIG. 10.-Higher magnification of upper lobe of Fig. 9 ( $\times 1.9)$.

lobular emphysema which is becoming confluent in the posterior apical segment, where rows of dilated bronchioles (dark) are separated by bands of less affected lung (lighter) near septa and septal veins. The lighter areas are the only surviving foci of relatively normal lung. The details of the upper lobe are shown in Fig. 12. The bulla in the anterior segment of the upper lobe may be either a superficial exaggeration of centrilobular disease or a separate phenomenon, possibly best described for the present as superficial emphysema. In the lower lobe (Fig. 11) there are several types of change. There is centrilobular emphysema in the middle zone. There is superficial emphysema posteriorly. There is some fine feathery localized diffuse emphysema (not clearly visible in the photograph) in the upper part of the posterior basal segment ; it is similar to that in Fig. 14, and probably a different or more marked variety of post-inflammatory change. In the lower part of the posterior basal segment there is post-inflamma- tory fibrosis with honeycombing. Much of the anterior basal segment shows diffuse emphysema.

An uncommon mixed pattern is shown in Fig. 13. It is from the right lung of a man of 76 who had chronic bronchitis for a "few years," and died of carcinoma of the oesophagus. There was centrilobular emphysema of much of the upper lobe and of the upper parts of the middle and lower lobes. Two types of lesion can be seen in the centre of the upper lobe (Fig. 13). In addition to centrilobular emphysema, the periphery of some lobules appears to have been stretched away from the thickened fibrous septa and associated septal veins, leaving thin filaments to bridge the gaps. A term which would seem to describe this appearance best is " paraseptal emphysema." Gough (1952) described bullae along blood vessels, bronchi, and septa. He attributed them to the expanding force of respiration which seemed to pull the degenerate lung substance from its framework. 


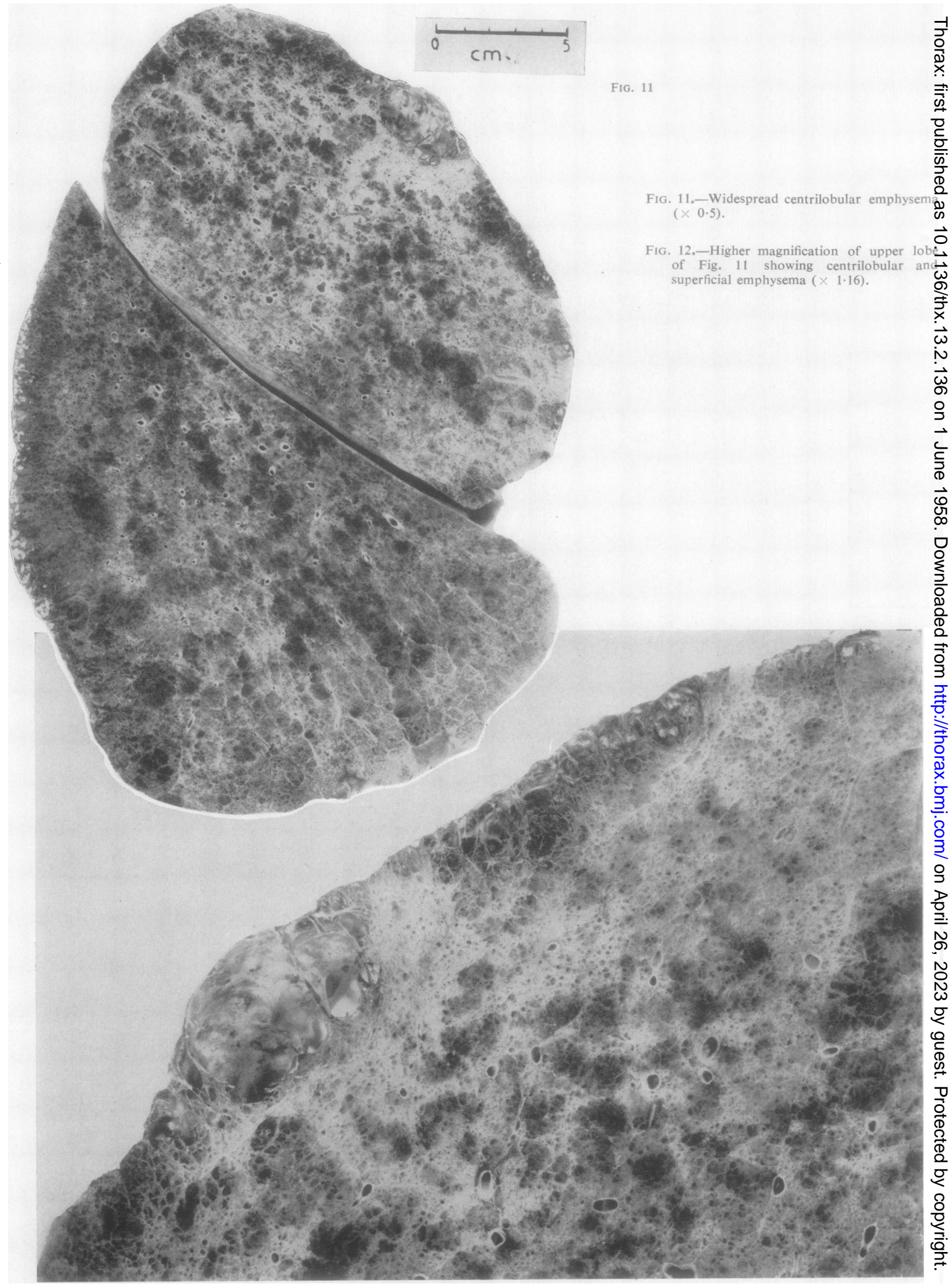

Fio. 12 


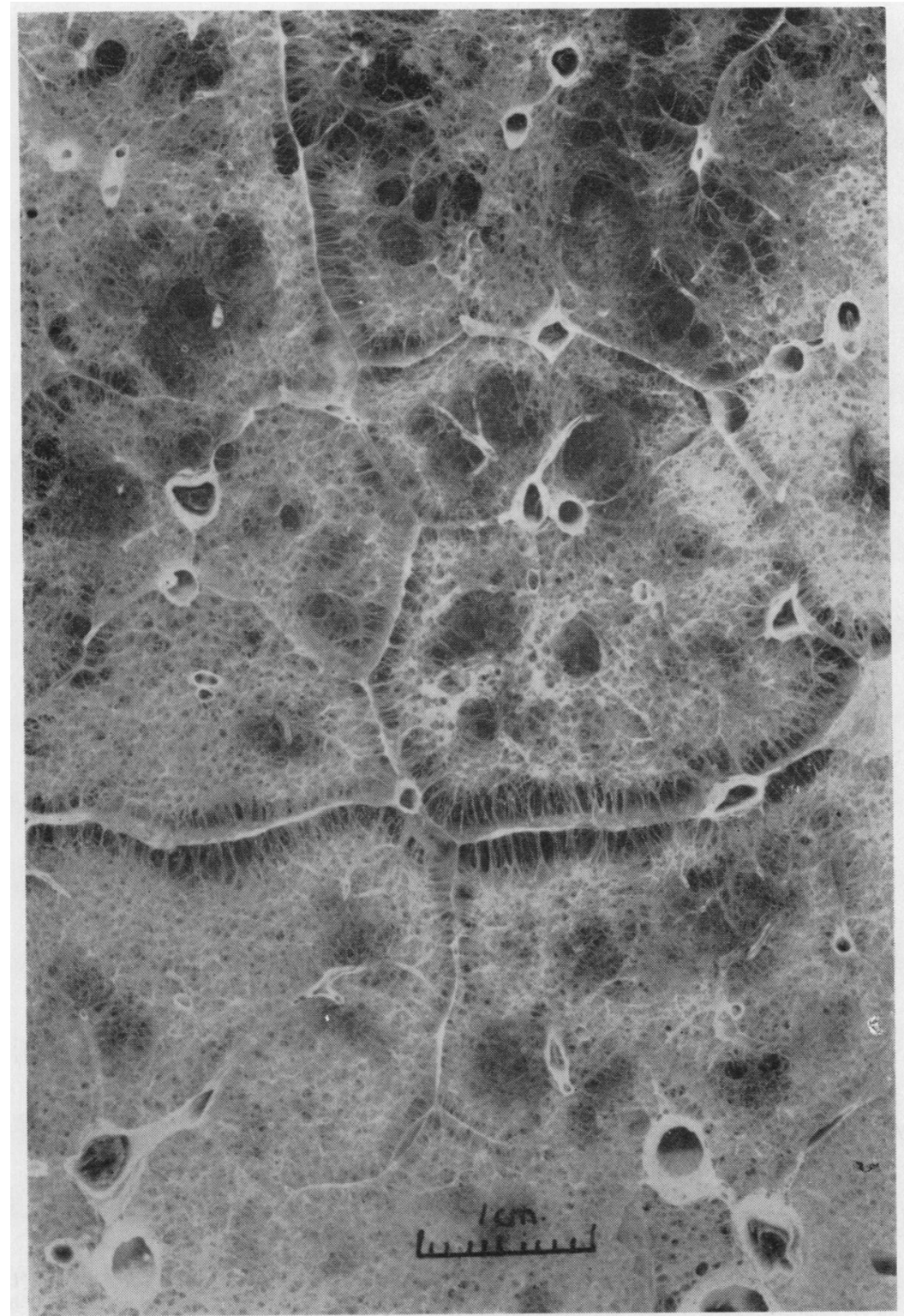

FIG. 13.-Centrilobular with paraseptal emphysema $(\times 2 \cdot 15)$.

Centrilobular emphysema is probably the consequence of centrilobular inflammation. If a localized wedge of lung is inflamed, the consequence may be localized emphysema, as in Fig. 14. This is the anterior segment of the right upper lobe of a woman of 80 years who had no sign of chronic bronchitis at any time, but had a febrile winter illness two years before death. She died of carcinoma of the pancreas with metastases, and the lung was taken as a control specimen. The photograph shows two paler areas (between arrows) of characteristic localized diffuse emphysema associated with bronchiectasis. There are some (dark) foci of localized centrilobular emphysema in the neighbourhood, but elsewhere the lung is normal.

With recurrent attacks of pulmonary inflammation of this type a number of such localized areas may accumulate, and may be associated with chronic bronchitis clinically. Such was the case 
present $(\times 1.06)$.

illustrated in Fig. 15, which was from a man of 75 who had had three attacks of pneumonia, 47, seven, and two years before dying from carcinoma of the prostate. He had had chronic bronchitis for some years. The photograph shows scarring in several areas of the left lung (sometimes with bronchiectasis), and about each scar is a zone of localized centrilobular emphysema. The endresult is almost equivalent in extent to a widespread centrilobular emphysema.

In conclusion, the lungs of established chronic bronchitics very often show centrilobular emphysema (and occasionally diffuse emphysema). The degree and extent of emphysema in control material were minimal. Centrilobular emphysema was usually widespread, but sometimes localized areas or accentuations were seen about scars following pneumonia or tuberculosis. Some cases showed bronchiectasis as well. The emphysematous lesions are revealed easily in specimens by the methods described.
A series of lungs from patients with chronic bronchitis is being collected and examined for emphysema. A number of the cases of wellestablished chronic bronchitis examined so far have shown widespread centrilobular emphysema, while lungs of non-bronchitics showed, at most, very mild grades in occasional scattered lobules.

Centrilobular emphysema has been missed until recent years because it is difficult to see in collapsed lungs at necropsy. It is best studied in formalin-distended lungs, but these distort if allowed to float in fixative. A method is described which overcomes shrinkage and distortion by maintaining the intrabronchial pressure at 10 in $(25 \mathrm{~cm}$.) of fixative for days or weeks at a time.

The translucency of sliced lung tissue has been overcome by barium sulphate impregnation. The resulting opacity of the finest structures allows reliable observations to be made by the naked eye 


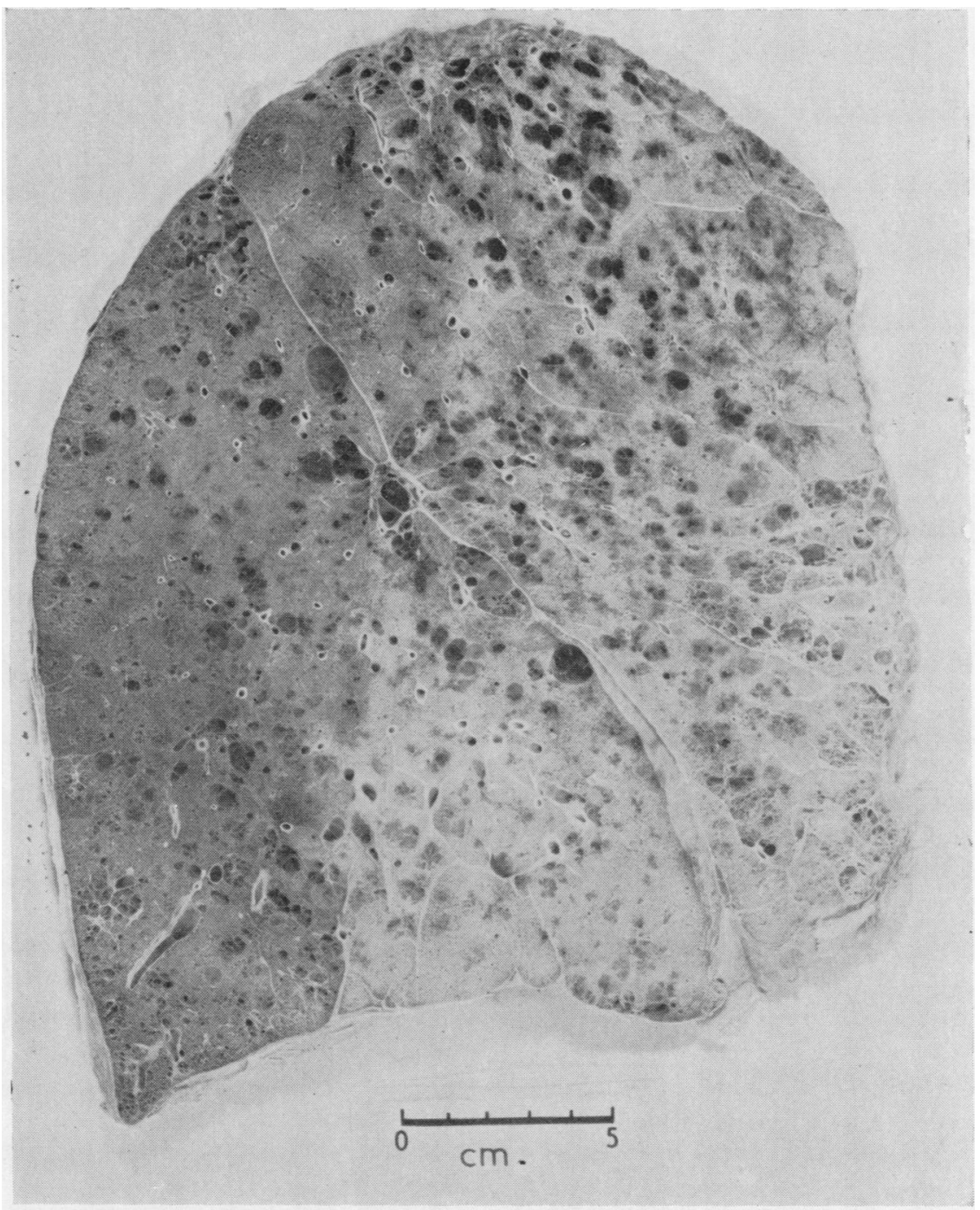

Fig. 15.-Localized centrilobular emphysema near many scars $(\times 0.5)$.

and fine details of air spaces can be studied by the dissecting microscope very satisfactorily. Some indication of this is given by the accompanying illustrations. A classification is described.

I wish to thank Professor C. V. Harrison for his interest and advice. I also wish to thank Mr. J. G. Griffin for technical help, Mr. W. H. Brackenbury and Mr. I. T. Hinton for the photographs, and Mr. F. G. Saunders for drawing Fig. 1.

\section{REFERENCES}

Cunningham, G. J., and Miller, J. W. (1952). Thorax, 7, 170. Fletcher, C. M. (1952). Proc. roy. Soc. Med., 45, 577. Gough, J. (1940). J. Path. Bact., 51, 277.
Gough, J. (1957). Personal communication.

and Wentworth,J. E. (1948). Proc.9th int. Congr. industr. Med., London, p. 661. Wright, Bristol.

Harrison, C. V. (1955). Personal communication.

Hoppleston, A. G. (1947). J. Path. Bact., 59, 453.

Loopold, J. G., and Gough, J. (1957). Thorax, 12, 219.

McLean, K. H. (1956a). Aust. Ann. Med., 5, 73.

(1956b). Ibid., 5, 254.

(1957a). Ibid., 6, 29.

(1957b). Ibid., 6, 124.

Oswald, N. C., Harold, J. T., and Martin, W. J. (1953). Lancet,

Reid, L. M. (1954). Ibid., 1, 275.

(1955). Thorax, 10, 199 .

(1956). Proc. roy. Soc. Med., 49, 771.

Scadding, J. G. (1952). In Diseases of the Chest, Vol. I, p. 55, by Marshall, Sir G., and Perry, K. M. A. Butterworth, London. Silver, A. W., Kirklin, J. W., Harris, L. E., and McDonald, J. R. (1956). Dis. Chest, 30, 456.

Spain, D. M., and Kaufman, G. (1953). Amer. Rev. Tuberc., 68, 24. Tompsett, D. H. (1952). Thorax, 7, 78 . 